\title{
Calibration and testing of the Planck-LFI QM instrument
}

A. Mennella ${ }^{a}$, B. Aja ${ }^{b}$, E. Artal $^{b}$, M. Balasini $^{c}$, G. Baldan $^{c}$, P. Battaglia $^{c}$, T. Bernardino $^{d}$, M. Bersanelli ${ }^{a}$, E. Blackhurst ${ }^{e}$, L. Boschini $^{c}$, C. Burigana ${ }^{f}$, R.C. Butler ${ }^{f}$, B. Cappellini ${ }^{a}$,

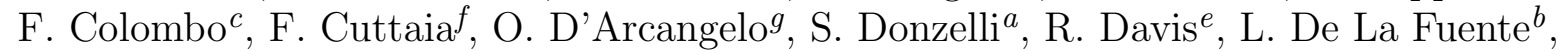
F. Ferrari ${ }^{c}$, L. Figini ${ }^{g}$, S. Fogliani ${ }^{h}$, C. Franceschet ${ }^{c}$, E. Franceschi ${ }^{f}$, T. Gaier ${ }^{i}$, S. Galeotta ${ }^{j}$, S. Garavaglia ${ }^{g}$, A. Gregorio ${ }^{h}$, M. Guerrini $^{h}$, R. Hoyland ${ }^{k}$, N. Hughes ${ }^{l}$, P. Jukkala ${ }^{l}$, D. Kettle ${ }^{e}$, M. Laaninen ${ }^{l}$, P.M. Lapolla ${ }^{c}$, D. Lawson $^{e}$, R. Leonardi ${ }^{m}$, P. Leutenegger ${ }^{c}$, G. Mari ${ }^{a}$, P. Meinhold ${ }^{m}$, M. Miccolis ${ }^{c}$, D. Maino ${ }^{a}$, M. Malaspina ${ }^{f}$, N. Mandolesi ${ }^{f}$, M. Maris ${ }^{h}$,

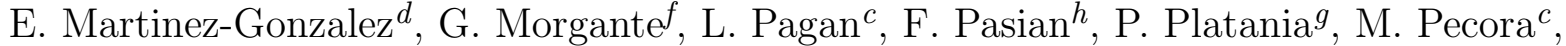

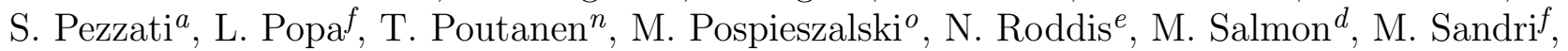

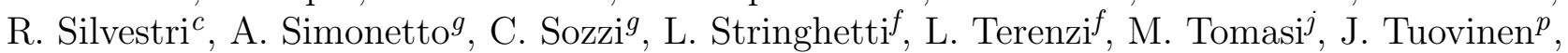

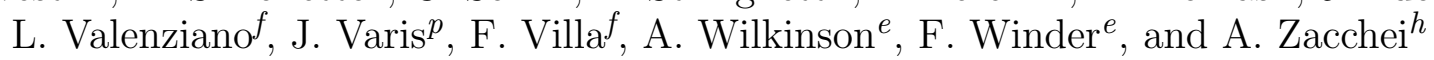

${ }^{a}$ Dipartimento di Fisica, Università degli studi di Milano, Milano, Italy

${ }^{b}$ Departamento de Ingeniería de Comunicaciones, Universidad de Cantabria, Santander, Spain

${ }^{c}$ Alcatel Alenia Spazio, Vimodrone, Milano, Italy

${ }^{d}$ Instituto de Fisica de Cantabria, Universidad de Cantabria, Santander, Spain

${ }^{e}$ Jodrell Bank Observatory, Jodrell Bank, U.K.

fIASF - Sezione di Bologna, INAF, Bologna, Italy

${ }^{g}$ Istituto di Fisica del Plasma, CNR, Milano, Italy

${ }^{h}$ Osservatorio Astronomico di Trieste, INAF, Italy

${ }^{i}$ Jet Propulsion Laboratories, Pasadena, United States

${ }^{j}$ IASF - Sezione di Milano, INAF, Milano, Italy

${ }^{k}$ Instituto de Astrofisica de Canarias, Tenerife, Spain

${ }^{l}$ Elektrobit Microwave Oy, Kauniainen, Finland

${ }^{m}$ Physics Department, University of California at Santa Barbara, USA

${ }^{n}$ Department of Physical Sciences, University of Helsinki, Helsinki, Finland

${ }^{\circ}$ NRAO, Charlotsville, USA

${ }^{p}$ MilliLab, VTT Information Technology, Espoo, Finland

\begin{abstract}
In this paper we present the test results of the qualification model (QM) of the LFI instrument, which is being developed as part of the ESA Planck satellite. In particular we discuss the calibration plan which has defined the main requirements of the radiometric tests and of the experimental setups. Then we describe how these requirements have been implemented in the custom-developed cryo-facilities and present the main results. We conclude with a discussion of the lessons learned for the testing of the LFI Flight Model (FM).
\end{abstract}

Keywords: Cosmic microwave background, differential receivers, space experiments

\section{INTRODUCTION}

The ESA Planck mission is the third generation space mission dedicated to the measurement of CMB anisotropies and is designed to fully extract the cosmological information contained in CMB anisotropy by setting angular resolution, spectral coverage and sensitivity such that the power spectrum reconstruction will be limited by

Send correspondence to Aniello Mennella

E-mail: aniello.mennella@fisica.unimi.it, Telephone: 390250317275

Space Telescopes and Instrumentation I: Optical, Infrared, and Millimeter, edited by John C. Mather, Howard A. MacEwen, Mattheus W.M. de Graauw,

Proc. of SPIE Vol. 6265, 62650G, (2006) -0277-786X/06/\$15 - doi: 10.1117/12.669408

Proc. of SPIE Vol. 6265 62650G-1 
unavoidable cosmic variance and astrophysical foregrounds. Planck will also provide a precise measurement of the TE correlation and of the E-mode polarisation power spectrum and possibly offer a first B-mode detection. ${ }^{1}$

The angular resolution is obtained with a $1.5 \mathrm{~m}$ aperture primary mirror, while the optimal optical response is offered by a combination of an off-axis dual reflector aplanatic telescope and dual-profiled corrugated feed horns in the focal plane to maximise beam symmetry and minimise sidelobe pick-up. The most effective removal of astrophysical foregrounds will be guaranteed by a wide frequency range, spanning from $30 \mathrm{GHz}$ to $\sim 900 \mathrm{GHz}$, a range obtained implementing different detector technologies, i.e. High Electrom Mobility Transistor (HEMT) radiometric receivers for the frequency range $30-70 \mathrm{GHz}$ (the LFI instrument ${ }^{2}$ ) and bolometric detectors for the frequency range 100-900 GHz (the HFI instrument ${ }^{3}$ ). The required sensitivity will be reached by a combination of wide-band components (20\% of the centre frequency for LFI, 30-40\% for HFI), long integration times (14 months of nominal mission time) and multiple cryogenically-cooled detectors. In particular the front-end amplifiers of the LFI instrument will be actively cooled at $\sim 20 \mathrm{~K}$, while the HFI bolometers will operate at $\sim 0.1 \mathrm{~K}$.

The core of this paper is dedicated to the discussion of the main results obtained during the calibration activity of the LFI Qualification Model (LFI-QM), which completed during 2005 with the testing of the individual radiometers (RCA, Radiometer Chain Assembly) and of the integrated instrument (RAA, Radiometric Array Assembly) performed at the laboratories of Alcatel-Alenia Space in Vimodrone (Milan). In Sect. 5 we summarise the main results comparing instrumental performances measured at RCA and RAA levels and discuss the main limitations and sources of uncertainty. Several lessons have been learned from the caibration activity of the LFI-QM; these lessons and guidelines (summarised in Sect. 6) constitute now the foundation of the now-ongoing testing phase of the LFI flight model.

\section{THE LFI INSTRUMENT}

The Low Frequency Instrument (LFI) is an array of coherent, differential radiometers operating in three frequency bands centred at 30, 44 and $70 \mathrm{GHz}$. The LFI receivers are based on cryogenic InP HEMT amplifiers actively cooled at $\sim 20 \mathrm{~K}$ by the Planck Sorption cooler. ${ }^{4}$ To minimise power dissipation in the focal plane the radiometers are split into two sub-assemblies connected by a set of custom designed waveguides.

The radiometers use a pseudo-correlation scheme to suppress $1 / f$ noise induced by amplifier gain and noise temperature fluctuations. ${ }^{5}$ In each radiometric chain the radiation entering a corrugated feed-horn is separated by an OrthoMode Transducer (OMT) into two perpendicular linearly polarised components that propagate independently through two parallel radiometers, in which a cryogenically cooled $20 \mathrm{~K}$ front-end is connected to a $300 \mathrm{~K}$ back end by means of composite waveguides (see Fig. 1, left panel).

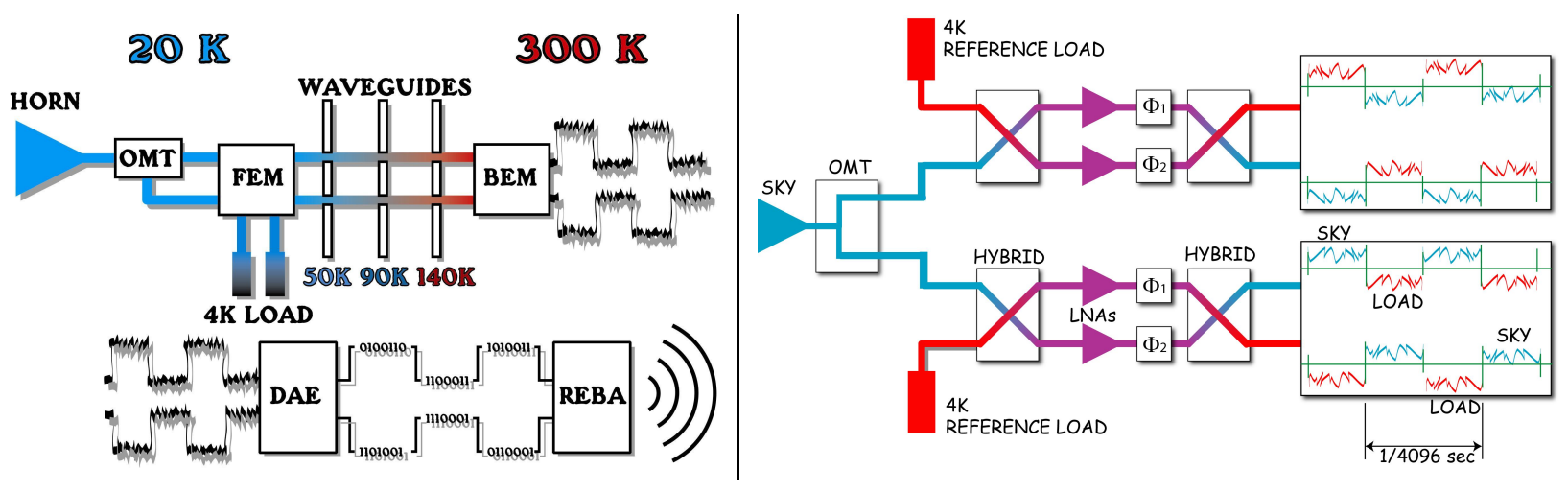

Figure 1. Left: a scheme of the Planck-LFI pseudo-correlation receiver. Each receiver is split into a $\sim 20 \mathrm{~K}$ front end and a $\sim 300 \mathrm{~K}$ back end connected by composite waveguides. Right: details of the pseudo-correlation architecture implemented in the receiver front end.

In each front-end (see Fig. 1, right panel), the sky signal and the signal from a stable reference load at $\sim 4 \mathrm{~K}$ (provided by the HFI $4 \mathrm{~K}$ cooling stage) are coupled to cryogenic low-noise HEMT amplifiers via a $180^{\circ}$ hybrid. 
One of the two signals then runs through a switch that applies a phase shift which oscillates between 0 and $180^{\circ}$ at a frequency of $4096 \mathrm{~Hz}$. The signals are then recombined by a second $180^{\circ}$ hybrid coupler, producing an output which is a sequence of signals alternating at twice the phase switch frequency. In the back-end the RF signals are further amplified, filtered by a low-pass filter and then detected. After detection, the data acquisition electronics (DAE in Fig. 1) integrates and digitises the voltage output which is then downsampled, requantised and compressed in a second electronics unit (REBA in Fig. 1) before sending to ground.

The differential measurement is taken on ground by differencing the sky and reference signals using a gain modulation factor $^{6}$ which compensates the residual input offset $(\lesssim 2 \mathrm{~K}$ in nominal conditions) and greatly improves the stability of the measured signal.

In Fig. 2 we show a 3D drawing (left panel) and a real picture of the LFI QM instrument at the end of the integration before the start of the test campaign. In the 3D drawing we can see the HFI integrated with the LFI at the focal plane centre. Two support structures (one in the upper part and the other in the lower part of the instrument) are also visible; these structures have been installed to increase the mechanical strength of the LFI waveguides, which are about $1.5 \mathrm{~m}$ in length. The interfaces with the three $\mathrm{V}$-grooves (conical-shaped thermal radiators which dissipate the heat coming from the warm satellite service module) are also noticeable in the figure.
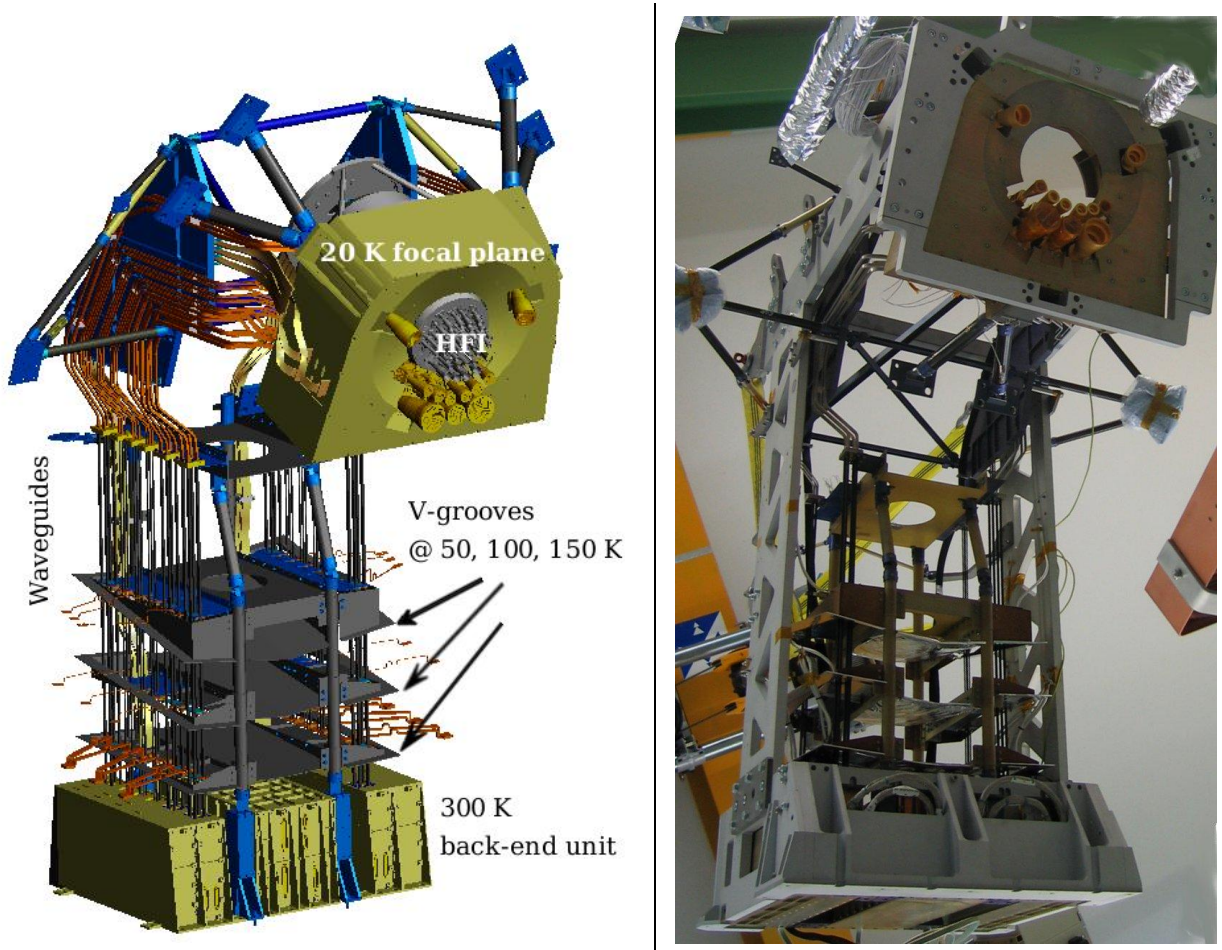

Figure 2. The Planck-LFI instrument. Left: 3D drawing; notice the presence of the HFI feed horns in the central part of the focal plane. Right: the LFI-QM before the start of the test campaign.

\section{THE LFI CALIBRATION PLAN}

The Planck-LFI is a complex, modular instrument in which several components (e.g. feed-horns, OMTs, individual low noise amplifiers, waveguides, etc.) have been developed and tested before their integration into higher level subassemblies to characterise functionality and performance.

Calibration and testing is therefore performed at all integration levels up to the satellite, and even in-flight, during the commissioning phase. Photometric and beam calibrations, to be used during data analysis, will be 
repeated in flight during nominal operations exploiting the well-known CMB dipole ${ }^{7}$ and the scanning of planets like Jupiter and Saturn. ${ }^{8}$ In Table 1 we summarise the tests that have been performed on single receivers and on the integrated instrument. ${ }^{9}$ Details about analytical and numerical methods used for analysing these data can be found in $[10,11]$.

Table 1. List of calibration and performance tests performed at the QM RCA and RAA levels.

\begin{tabular}{|l|l|l|}
\hline \multirow{5}{*}{ Functionality } & Room temperature & Verification of phase switch and low noise amplifier functionality \\
\cline { 2 - 3 } Tuning & Cryogenic temperature & $\begin{array}{l}\text { Tuning of phase switch bias currents in order to optimise amplitude } \\
\text { balance in the two phase switch states }\end{array}$ \\
\cline { 2 - 3 } & Phase switch bias currents & $\begin{array}{l}\text { Tuning of amplifier gate 1 voltage for optimal noise temperature } \\
\text { and of gate 2 voltage for optimal gain balance (isolation) }\end{array}$ \\
\cline { 2 - 3 } & REBA parameters (RAA only) & Second quantisation, compression rate. \\
\hline \multirow{5}{*}{ Performance } & Basic radiometric parameters & $\begin{array}{l}\text { Noise temperature, calibration constant, linearity, input offset. } \\
\text { Measured from average values of voltage outputs and input tem- } \\
\text { peratures. The measurement requires acquisition of receiver data } \\
\text { at different values of the input sky (or reference) load temperatures. }\end{array}$ \\
\cline { 2 - 3 } & Noise properties & $\begin{array}{l}\text { Noise effective bandwidth, white noise, 1/f noise knee frequency } \\
\text { and slope. Measured from data measured in stable input tempera- } \\
\text { ture conditions }\end{array}$ \\
\cline { 2 - 3 } & Susceptibility & $\begin{array}{l}\text { Susceptibility to boundary temperature and front-end bias voltage } \\
\text { variations. }\end{array}$ \\
\cline { 2 - 3 } & Spectral response (RCA only) & $\begin{array}{l}\text { The measurement is performed by injecting into the sky-load a } \\
\text { monochromatic wave sweeping through the receiver bandpass and } \\
\text { recording the voltage output }\end{array}$ \\
\hline
\end{tabular}

\section{TEST SETUP}

\subsection{The RCA cryogenic test setup}

In left panel of Fig. 3 we show the cryogenic chamber bench with a $30 \mathrm{GHz}$ RCA just before the installation of the sky and reference loads and of thermal and electrical connections. In the right panel we show a detail of the receiver feed horn pointing in the inner of the sky load, an ECCOSORB cavity designed to provide a return loss better than $30 \mathrm{~dB}$.
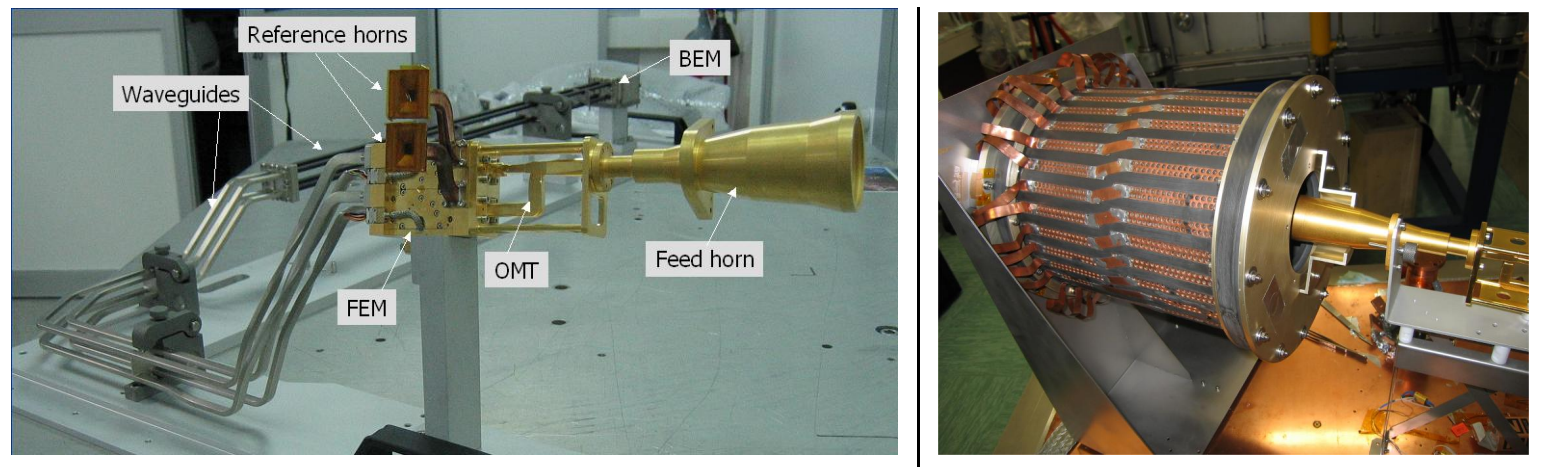

Figure 3. Left: the RCA cryogenic test setup with a $30 \mathrm{GHz}$ receiver before the installation of the sky and reference loads and before being electrically connected and thermally set up for testing. Right: detail of the feed horn pointing in the inner of the sky load.

During testing the chamber is set at a pressure of $\sim 5 \times 10^{-5}$ mbar with the following temperature stages: a $40 \mathrm{~K}$ plate and shroud providing the radiative environment to the $20 \mathrm{~K}$ front-end module, a $20 \mathrm{~K}$ stage 
where the receiver front-end is attached to, two independently controlled $4 \mathrm{~K}$ stages connecting the input loads (sky and reference), two thermal interfaces with waveguides at $\sim 22 \mathrm{~K}$ and $\sim 60 \mathrm{~K}$, respectively, and a $300 \mathrm{~K}$ heater-controlled stage connected to the receiver back-end module.

Temperatures are provided by three two-stage coolers: an ARS $4 \mathrm{~K}$ cooler with $0.8 \mathrm{~W}$ cooling power, a Leybold $20 \mathrm{~K}$ cooler with $5 \mathrm{~W}$ cooling power and a CTI $20 \mathrm{~K}$ cooler with $10 \mathrm{~W}$ cooling power. Temperatures are monitored by 12 sensors (Cernox resistances and silicon diodes); in particular the sky load temperature (which is crucial for the calculation of many receiver parameters) is monitored by two high sensitivity $(\sim 0.1 \mathrm{mK}$ at $4 \mathrm{~K})$ sensors, one placed on the centre of the back metal plate, and the other on the side of the ECCOSORB structure. PID temperature controls placed on all stages (apart from the $40 \mathrm{~K}$ plate) have allowed to reach temperature stabilities of the order of few $\mathrm{mK}$ on time scales of $\sim 1$ hour,

A cryo-chamber with similar performances and able to host two complete RCAs (which can be tested at the same time) has been set up at the Elektrobit laboratories for testing the $70 \mathrm{GHz}$ RCAs (see Fig. 4).
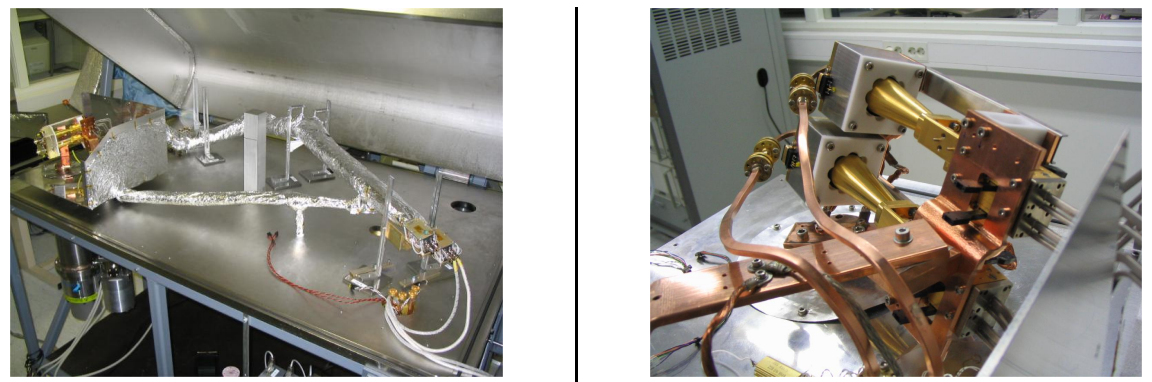

Figure 4. The facility for the $70 \mathrm{GHz}$ RCA testing (left) and a detail of two horns coupled with the sky loads (right).

\subsection{The RAA cryogenic test setup}

In Fig. 5 we show the RAA cryo-chamber with the LFI in place and ready for testing.
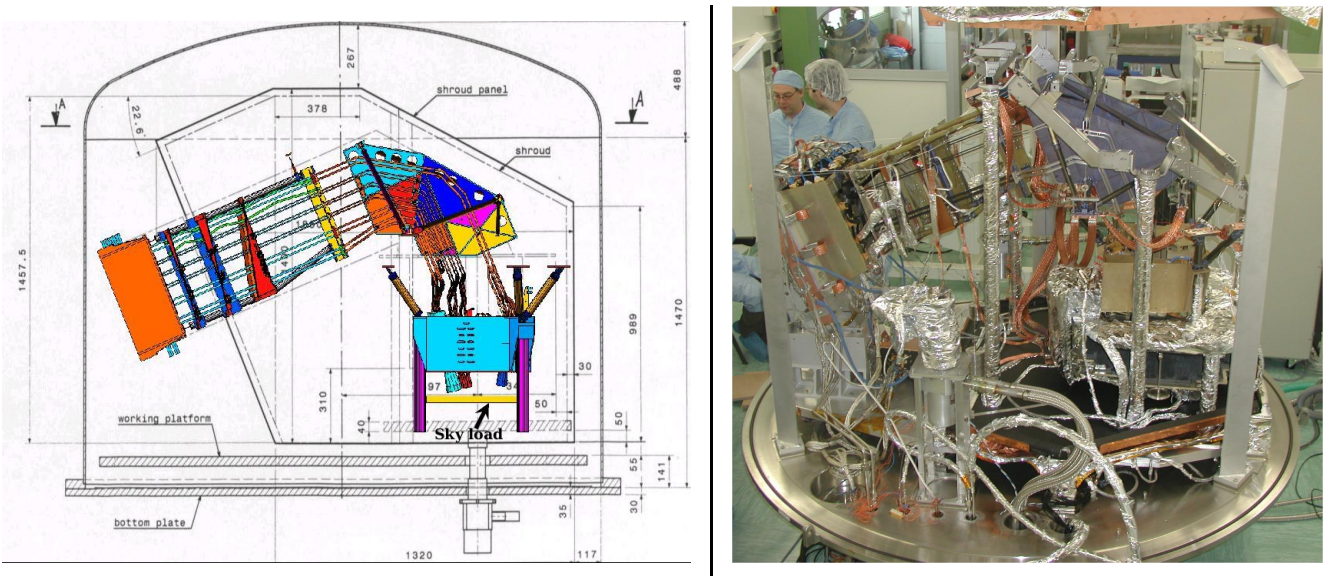

Figure 5. Left: drawing of the RAA cryo-chamber with the LFI in the testing position. Right: picture of the RAA cryo-facility with the LFI-QM ready for testing before the chamber closure.

The left panel shows a drawing of the chamber with the LFI looking down towards a layer of ECCOSORB pyramids constituting the sky load; in the right panel we can see the real facility with the LFI-QM ready for 
testing just before the chamber closure. The minimum achievable pressure is of the order of $2 \times 10^{-6}$ mbar with a pumpdown time of the order of 10-15 hours. The main thermal stages, all PID-controlled for temperature stability, are: a $40 \mathrm{~K}$ thermal tent around the focal plane unit providing the radiative environment, a $20 \mathrm{~K}$ stage connected to the focal plane unit, two $20 \mathrm{~K}$ stages connected to the input sky and reference loads, three thermal stages connected at the three V-groove interfaces at $\sim 50 \mathrm{~K}, \sim 96 \mathrm{~K}$ and $\sim 146 \mathrm{~K}$, respectively, a $300 \mathrm{~K}$ stage connected to the back-end unit and regulated via a heater and a thermostatic bath.

These temperatures have been reached using two single-stage coolers, a Cryomech AL200, with $35 \mathrm{~W}$ cooling power at $30 \mathrm{~K}$, and a Cryomech AL220, with $25 \mathrm{~W}$ cooling power at $20 \mathrm{~K}$.

Two different sets of diode temperature sensors have been implemented. One set for monitoring the cryochamber temperatures (12 on the thermal tent, two for each V-groove interface, two on the back-end unit, two on the front-end unit, two on the sky load and two on the reference load); the second set (composed by 12 sensors on the front-end unit and 10 on the back-end unit) is part of the instrument and sends temperature information directly in the instrument telemetry.

Reaching the required thermal performances has been more problematic in the RAA cryochamber: in particular a too-high thermal resistance of copper thermal straps and a problem in the cooling power of one of the $20 \mathrm{~K}$ coolers determined a focal plane temperature which was $\sim 5 \mathrm{~K}$ higher than the required $20 \mathrm{~K}$. Moreover the banck-end unit temperature was affected by a $\sim 24 \mathrm{hrs}$ period variation of few $\mathrm{K}$ that showed up as a spurious effect and required de-correlation of the receiver data before analysis. Both problems have been understood and addressed and will likely be solved for the flight model tests.

\section{MAIN RESULTS}

In this section we summarise the main results of the LFI-QM testing campaign by comparing the most important radiometric properties measured at RCA and RAA levels (i.e. noise temperature, isolation, white noise sensitivity, noise effective bandwidth, $1 / f$ noise knee frequency and slope).

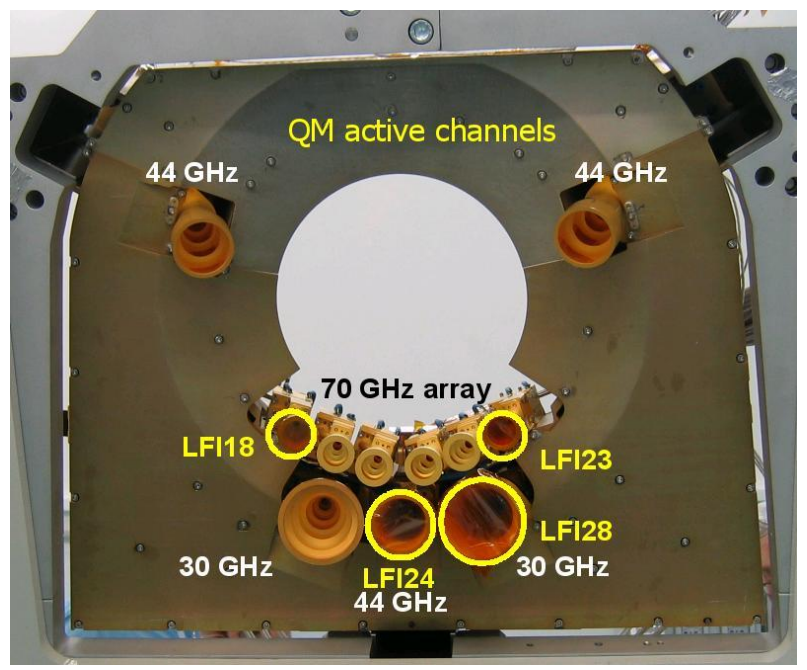

Figure 6. The focal plane unit of the LFI-QM. Circled horns represent the active channels during the test campaign. The other horns in the focal plane are thermally/mechanically representative dummies.

Before going into the details of the measured parameters we need to underline the following facts:

- the LFI-QM was meant to provide a prototype fully representative of the instrument functionality but not of the flight performances. The measured QM performance were typically a factor $\sim 2$ above the FM 
specifications and in line with the expectations based on sub-unit measurements. In the following discussion we do not make any detailed comparison between the measured performances and goal/requirement specifications;

- the main objective in the RAA tests was to verify the consistency with measurements performed at RCA level. This has not been a simple task and this exercise helped us identify the main limitations that have now been overcome for the upcoming flight model test;

- the LFI-QM is composed by a subset of the full radiometric array: one receiver at $30 \mathrm{GHz}$, one at $44 \mathrm{GHz}$ and two at $70 \mathrm{GHz}$. Fig. 6 shows the LFI focal plane with the real receivers that were tested (circled horns) and the remaining dummies. In this paper we quote results obtained for LFI28, LFI24 and LFI23. The 70 GHz LFI18 showed functionality problems during the RAA tests that prevented a full characterisation of its performances.

\section{1. $30 \mathrm{GHz}$ receiver results}

\subsubsection{Noise temperature and isolation}

Receiver noise temperature and isolation have been measured from a series of tests in which the sky load temperature was varied and the corresponding voltage output recorded. In the left panel of Fig. 7 we show the voltage $v s$ temperature diagram relative to one output diode for all datasets. In the right panel on the $y$ axis we plot the difference between the sky and reference voltage outputs, $\Delta V=V_{\text {sky }}-V_{\text {ref }}$, as a function of the difference between the sky and reference load temperatures, $\Delta T=T_{\text {sky }}-T_{\text {ref }}$. The plots show the good repeatability and linearity of the receiver response; this is particularly apparent in the right panel, where the differential plot removes the effects of any variations/instabilities common to the sky and reference load data.
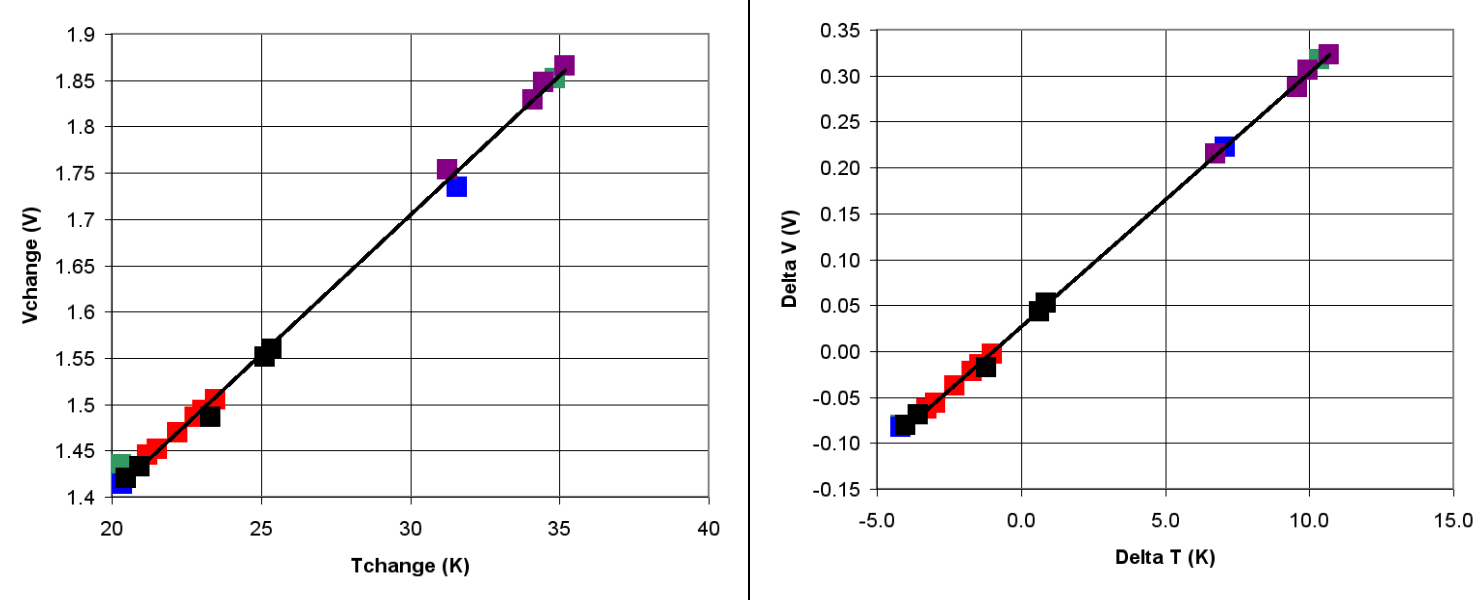

Figure 7. Left: voltage-temperature diagram for one channel output of the $30 \mathrm{GHz}$ LFI28 receiver. Right: $\Delta T-\Delta V$ diagram. The diagrams collects points acquired during different tests through all the campaign.

In Fig. 8 we show a comparison of the measured noise temperature (left panel) and isolation* (right panel) for the four output channels. The main source of uncertainty in the noise temperature measurement has been the choice of the sky load temperature sensor used in the calculation. The bottom and the top of the gray regions in the noise temperature plots represent the minimum and the maximum values obtained considering different sky load temperature sensors. To overcome this source of uncertainty we have installed, for the flight model tests, temperature sensors directly on the ECCOSORB pyramids facing the receiver feeds.

\footnotetext{
${ }^{*}$ In a test in which the sky load temperature is varied, the isolation is defined, for each receiver output, as the relative change in the reference voltage output compared to the total voltage output, in other terms: $I=\frac{\Delta V_{\text {ref }}}{\Delta V_{\text {sky }}+\Delta V_{\text {ref }}}$.
} 
The plots show that the noise temperature estimate was repeatable between RCA and RAA tests within the experimental uncertainty; on the other hand we have found a lower isolation in the RAA tests compared to the RCA, a mismatch that we have not been able to fully understand yet, considering that the uncertainty, in this case, was of the order of $\sim 1 \mathrm{~dB}$, thus less than the measured difference.
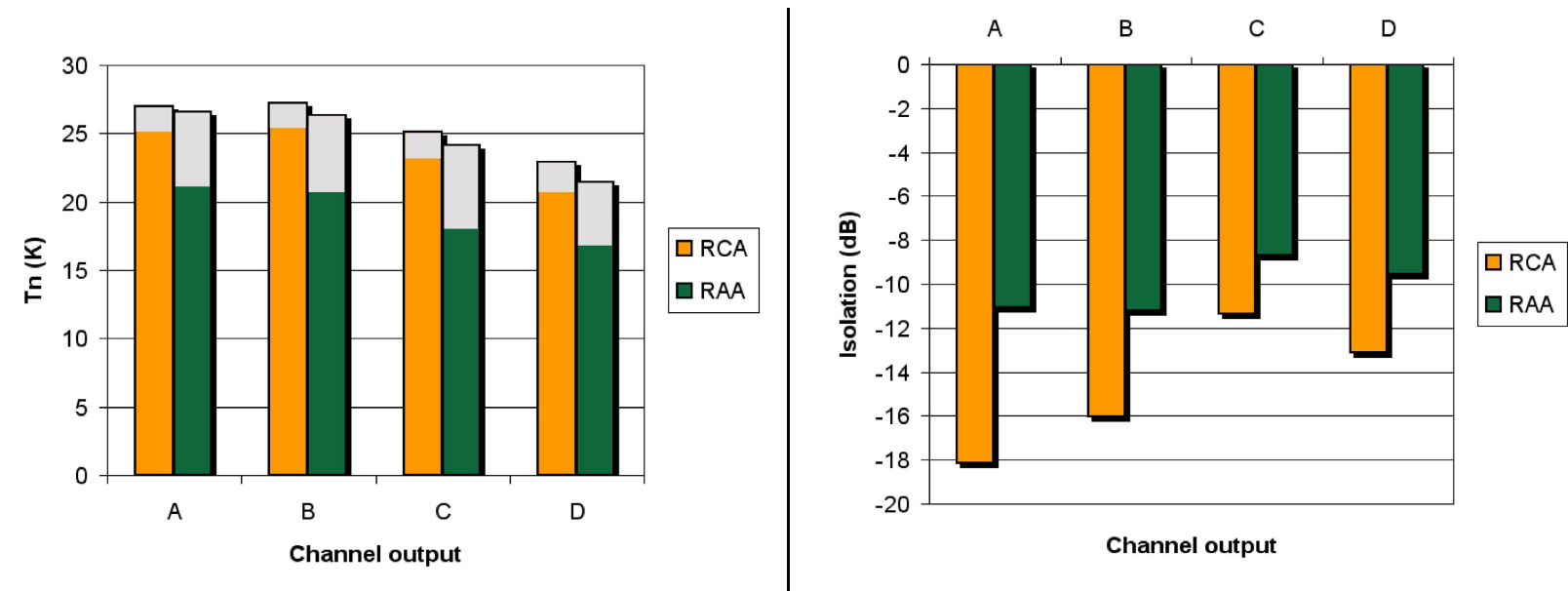

Figure 8. Basic parameters of the $30 \mathrm{GHz}$ LFI 28 receiver (RCA and RAA measurements). Left: noise temperature. Gray areas represent the measurement uncertainty, mainly driven by the uncertainty on the most representative sky load temperature sensor. Right: isolation. The uncertainty, in this case, is of $\sim 1 \mathrm{~dB}$ and it is not shown in the plot.

\subsubsection{Noise properties}

In this section we summarise the main noise properties, i.e. the white noise sensitivity, the noise effective bandwidth (derived from the white noise limit ${ }^{10,11}$ ) and the $1 / f$ knee frequency.
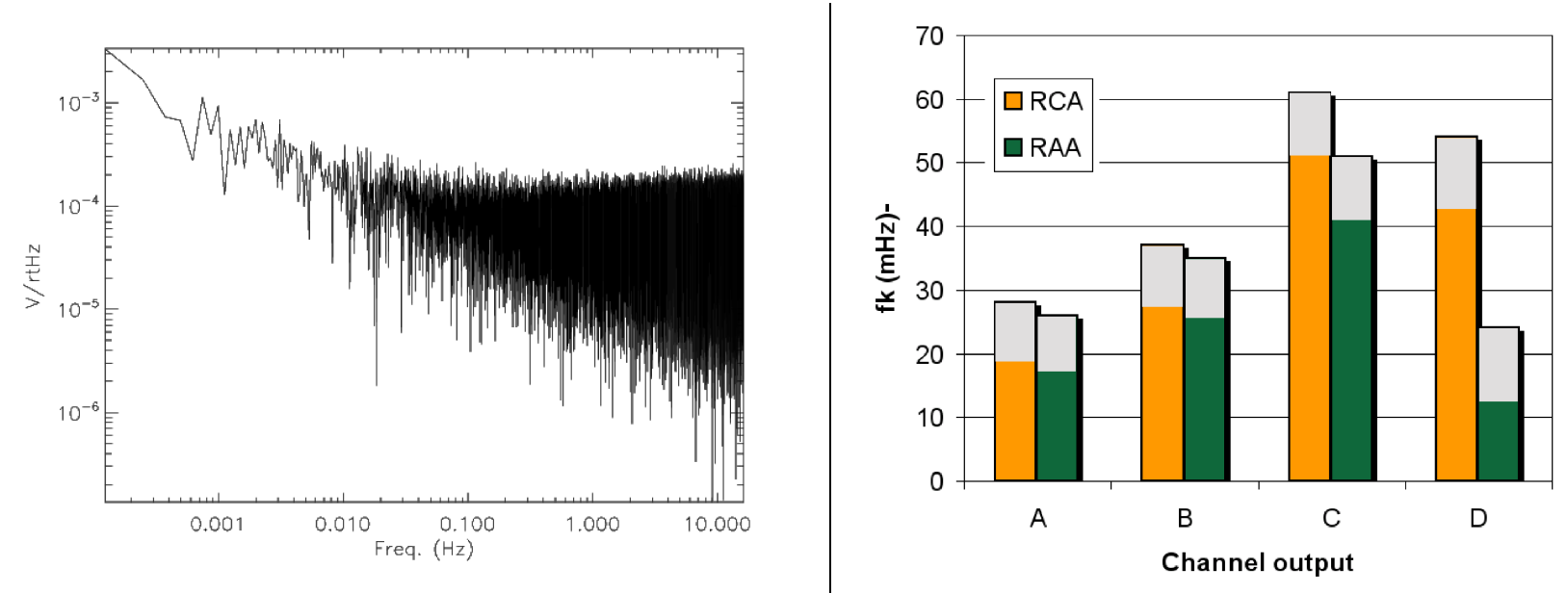

Figure 9. Left: amplitude noise spectrum of the differenced radiometric output (channel C). Right: comparison of knee frequencies determined at RCA and RAA levels.

For the LFI differential pseudo-correlation receivers the r.m.s. 1-second white noise is defined ${ }^{5}$ as $\Delta T_{\text {rms }}=$ $\sqrt{2}\left(T_{\text {sky }}+T_{\text {noise }}\right) / \sqrt{\beta}$ where $\beta$ represents the receiver bandwidth. The white noise measured from the noise 
spectrum also provides a measurement of the noise effective bandwidth, which is defined as: $\beta=2 V_{\mathrm{DC}}^{2} / \Delta V_{\mathrm{rms}}^{2}$, where $V_{\mathrm{DC}}$ represents the receiver $\mathrm{DC}$ voltage output.

In the left panel of Fig. 9 we show a typical amplitude noise spectral density plot of the differenced output from one of the four output channels; the white noise limit and the $1 / f$ tail with a knee frequency of $\sim 40 \mathrm{mHz}$ are apparent. In the right panel we plot the knee frequencies measured at RCA and RAA levels. The typical uncertainty in the knee frequency estimation is of the order of $\sim 10 \mathrm{mHz}$ and it is determined by the choice of parameters in the fitting procedure. As in the noise temperature plots the bottom and top of the gray areas represent the extrema in knee frequency calculated with different fitting parameters.

The results indicate a general agreement between RCA and RAA measurements, with the exception of channel D, which shows a discrepancy that we could not completely explain. However our results indicate that the differencing scheme of the LFI radiometers is effective in reducing the $1 / f$ noise amplifier instabilities at very low levels, compatible with the tight scientific requirements imposed by the mission $(\lesssim 50 \mathrm{mHz})$.

In Fig. 10 we summarise the main noise parameters measured during RCA and RAA tests. In the left panel we plot the white noise sensitivity in $\mathrm{mK} \times \sqrt{\mathrm{s}}$, i.e. after multiplication by the photometric constant (which is measured from data plotted in Fig. 7). While the error on the bare white noise in $m V \times \sqrt{\mathrm{s}}$ is of the order of few $\%$, the uncertainty on the final sensitivity is greater, of the order of $\sim 10-20 \%$ and is dominated by the uncertainty in the photometric calibration which is sensitive to the choice of the temperature sensor data used in the computation.

The difference shown in the figure between sensitivities measured at RCA and RAA levels, however, are caused by the different experimental conditions in the two cases, namely the sky load temperature that was $\sim 35 \mathrm{~K}$ in the RAA and $\sim 21 \mathrm{~K}$ in the RCA. Therefore in the centre graph we plot the ratio $\rho=\frac{\mathrm{WN}(\mathrm{RAA})}{\mathrm{WN}(\mathrm{RCA})} \frac{\left(T_{\mathrm{sky}}+T_{\mathrm{sys}}\right)(\mathrm{RCA})}{\left(T_{\mathrm{sky}}+T_{\mathrm{sys}}\right)(\mathrm{RAA})}$, i.e. the ratio of white noise limits over the ratio of the sums of sky and system temperatures; this ratio should obviously be close to 1 , and the results clearly indicate that the level of consistency is at the level of $\sim 10 \%$. The same level of consistency is obtained comparing the noise effective bandwidths derived by the white noise limit of the differenced data.
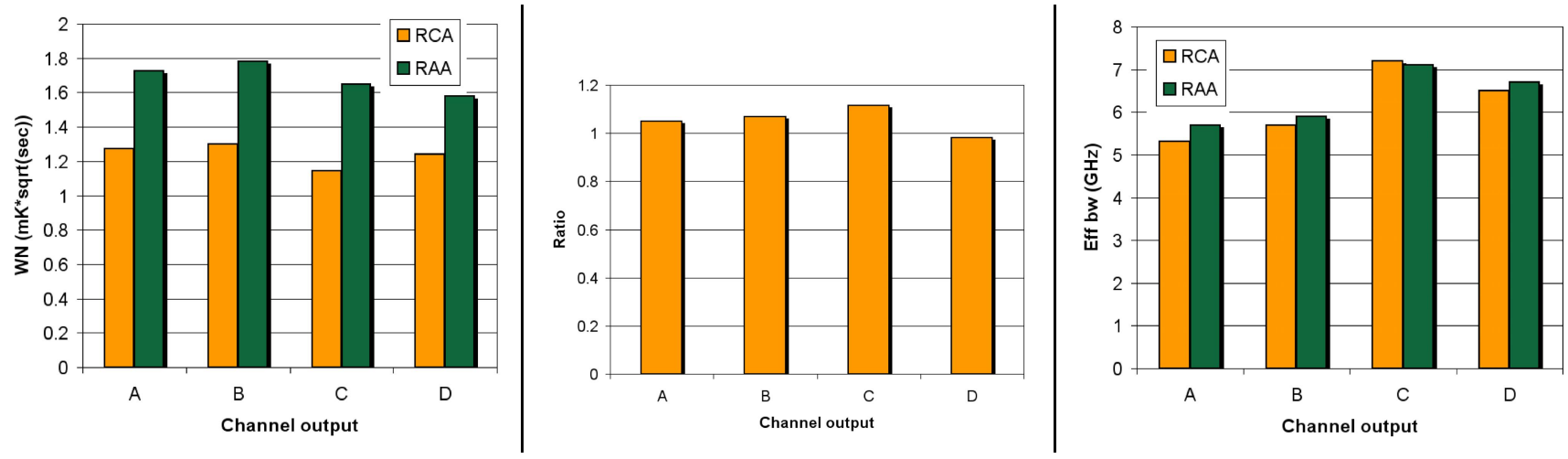

Figure 10. Noise properties of the $30 \mathrm{GHz}$ LFI28 receiver measured during RCA and RAA tests. Left: white noise sensitivity. Centre: ratio $\rho$ (see text for definition). Right: noise effective bandwidths.

\section{2. $44 \mathrm{GHz}$ receiver results}

\subsubsection{Noise temperature and isolation}

In Fig. 11 we summarise the basic receiver properties of the $44 \mathrm{GHz}$ receiver LFI24. The noise temperature, plotted in the left panel, shows a good match between RCA and RAA measurements for channels C and D, while it appears to be much higher in the RAA measurements for channels A and B. This apparently inconsistent results has been explained as the effect of a slightly non linear response at high input temperatures due to 
compression of the back-end RF amplifiers. In the flight model RCAs at $44 \mathrm{GHz}$ the non linear response has then been thoroughly characterised and taken into account in the calculation of the radiometric properties. Isolation measurements match at the level of $\sim 2 \mathrm{~dB}$ apart from channel $\mathrm{D}$, where the mismatch of $\sim 5 \mathrm{~dB}$ has not been completely understood yet.
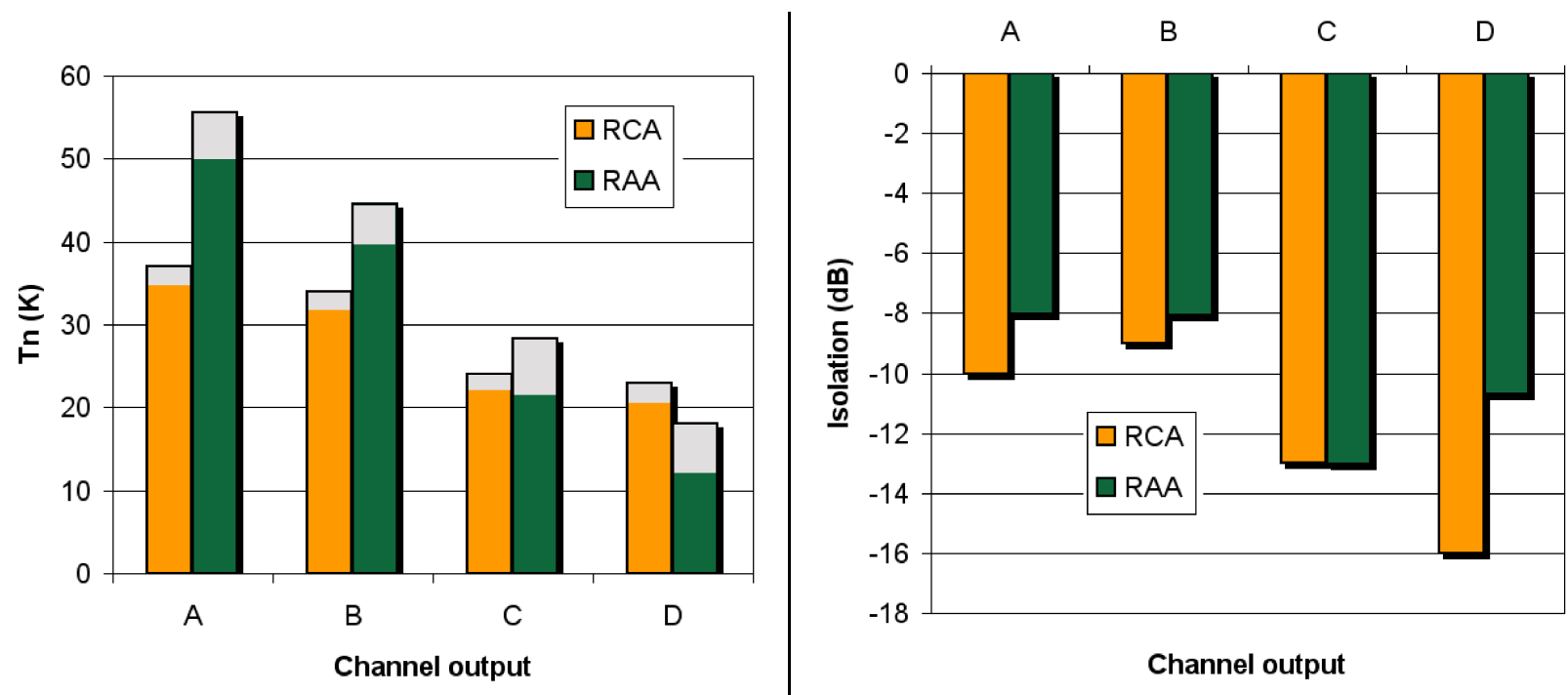

Figure 11. Basic receiver parameters of the $44 \mathrm{GHz}$ LFI24 receiver (RCA and RAA measurements). Left: noise temperature. Right: isolation.

\subsubsection{Noise properties}

In Fig. 12 we summarise the main $44 \mathrm{GHz}$ noise properties measured during the RAA tests.
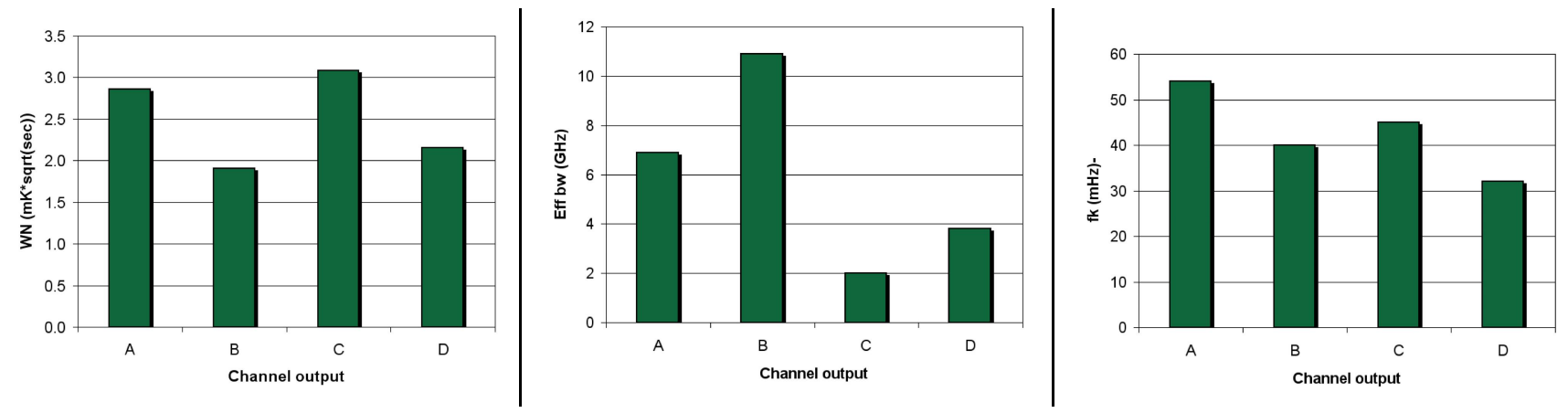

Figure 12. Noise properties measured during RAA tests. Left: white noise sensitivity. Centre: noise effective bandwidths. Right: $1 / f$ noise knee frequency.

In this case we could not make a comparison with RCA measurements as in this latter case the receiver noise was not resolved (i.e. it was dominated by the back-end acquisition noise) because of incorrect settings of the data acquisition gain stage that optimises the output voltage to the dynamic range of the ADC converter. This problem was recongnised only after the end of the RCA tests that could not be repeated because of schedule constraints. 
In the results reported in Fig. 12 the most noticeable are the very low bandwidths (compared to the required 8.8 $\mathrm{GHz}$ ) measured on channels $\mathrm{C}$ and $\mathrm{D}$. This was a well known problem caused by ripples in the BEM gain, a problem that has been solved in the flight models.

\section{3. $70 \mathrm{GHz}$ receiver results}

In the case of the $70 \mathrm{GHz}$ receiver the match between measurements performed at RCA and RAA levels has been particularly difficult, mainly because the $70 \mathrm{GHz}$ RCAs were tested in a very different configuration compared to the 30 and $44 \mathrm{GHz}$ RCAs. The former, in fact, have been tested in Finland at the Elektrobit laboratories, in a slightly different cryogenic environment, with representative waveguides (not the same that were used in the RAA tests) and with a totally different data acquisition and bias control system. Although we knew that this was not an ideal situation, schedule and resources constraints have forced us to follow a simpler, though not ideal, approach to the $70 \mathrm{GHz}$ RCA testing.

As an example of the level on incosistency we show in Fig. 13 the noise temperature and isolation calculated at RCA and RAA levels. These limitations have been overcome for the $70 \mathrm{GHz}$ flight RCAs that have been tested with the flight waveguides and with the same data acquisition and bias control system used also for the other frequencies and that is completely representative of the electronics used in the integrated instrument.
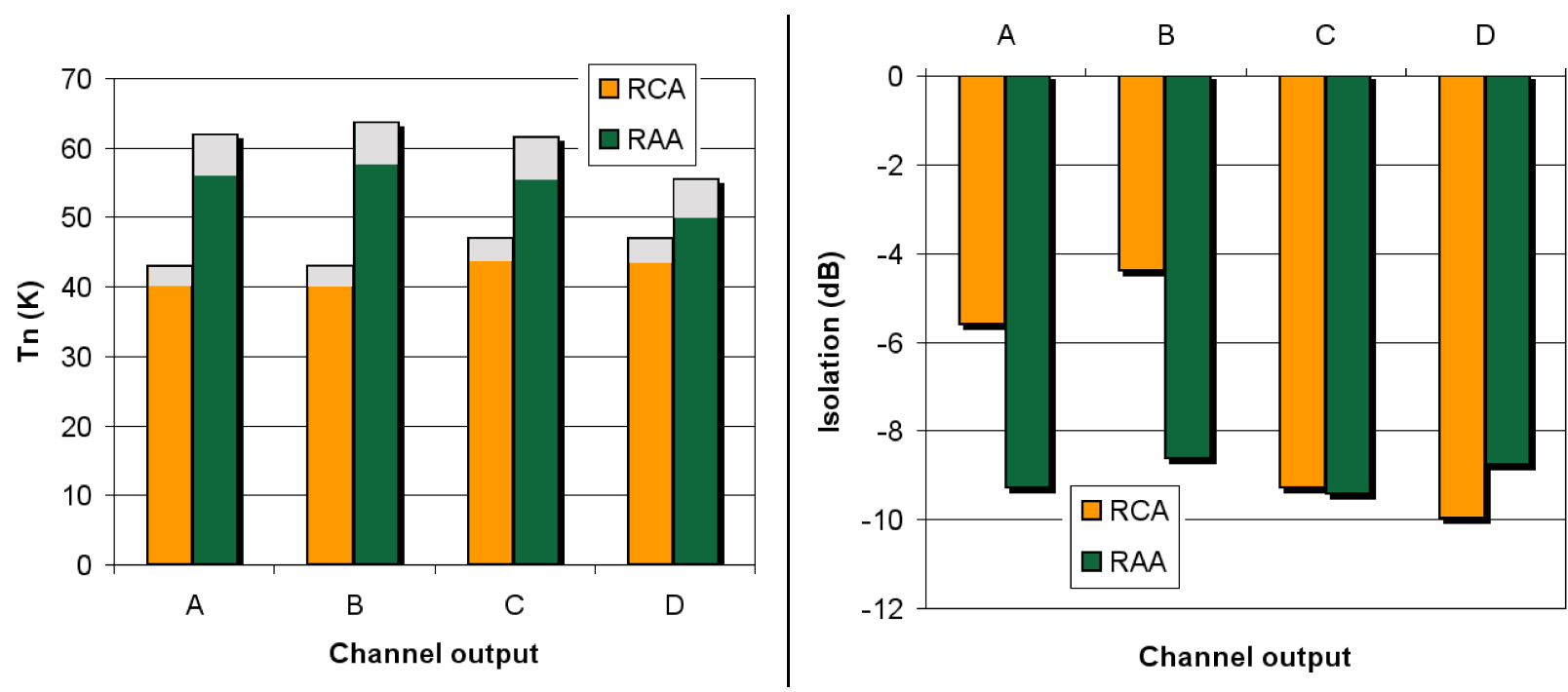

Figure 13. Basic receiver parameters of the $70 \mathrm{GHz}$ LFI23 receiver (RCA and RAA measurements). Left: noise temperature. Right: isolation.

\section{CONCLUSIONS AND LESSONS LEARNED}

Testing the LFI QM turned out to be a tough exercise, as for the first time several hardware and software sub-systems have been interfaced in a very complex cryogenic environment. Major achievements have been the definition of a consolidated test procedure and the development of a higher level of confidence in the various subsystems. This experience has shown the importance to have a common data acquisition and bias control setup, as well as a test plan and procedures overlapping as much as possible between RCA and RAA tests. Good reproducibility of results between RCA and RAA have been obtained at 30 and $44 \mathrm{GHz}$, in which commonalities were maximised, although also in these cases some mismatching results have not been fully clarified yet.

Important lessons concerning the test setup and the thermal environment have been also learned. In particular it is crucial that a thorough check of temperature stabilities at the various thermal stages and a careful tuning of the PID controllers are performed before the start of the radiometric tests. It is also of key importance a precise 
knowledge of the temperature that is actually "seen" by the sky and reference horns of the receiver; to minimise this kind of uncertainty we have increased the number of temperature sensors on the sky and reference loads, thus providing much more detailed information about the temperature distribution. Finally the test plan must foresee the same tests performed on the RCA and RAA with the same procedure and the same data analysis algorithms. This philosophy has been striclty followed in the flight model tests, which have been analysed using a common software specifically developed for this purpose. ${ }^{11}$

The benefits gained from these lessons have been already apparent to the LFI calibration team during the RCA flight model test campaign that is about to be concluded, although we are aware that understanding, testing and operating such a complex and highly performing instrument remains a high-level technical challenge that will continue well beyond the end of the LFI test campaign.

\section{REFERENCES}

1. J. Tauber, M. Bersanelli, J.-M. Lamarre, G. Efstathiou, C. Lawrence, F. Bouchet, E. Martinez-Gonzalez, S. Matarrese, D. Scott, M. White, N. Aghanim, M. Bartelmann, B. Partridge, R. Rowan-Robinson, J.-L. Puget, M. Giard, and R. Davis, "Planck - the scientific programme," Tech. Rep. ESA-SCI(2005)1, ESA, 2005.

2. A. Mennella, M. Bersanelli, B. Cappellini, D. Maino, P. Platania, S. Garavaglia, R. C. Butler, N. Mandolesi, F. Pasian, O. D'Arcangelo, A. Simonetto, and C. Sozzi, "The Low Frequency Instrument in the ESA PLANCK mission," in AIP Conf. Proc. 703: Plasmas in the Laboratory and in the Universe: New Insights and New Challenges, G. Bertin, D. Farina, and R. Pozzoli, eds., pp. 401-404, Apr. 2004.

3. J.-M. Lamarre, J. L. Puget, M. Piat, P. A. R. Ade, A. E. Lange, A. Benoit, P. De Bernardis, F. R. Bouchet, J. J. Bock, F. X. Desert, R. J. Emery, M. Giard, B. Maffei, J. A. Murphy, J.-P. Torre, R. Bhatia, R. V. Sudiwala, and V. Yourchenko, "Planck high-frequency instrument," in IR Space Telescopes and Instruments. Edited by John C. Mather . Proceedings of the SPIE, Volume 4850, pp. $730-739$ (2003)., J. C. Mather, ed., pp. 730-739, Mar. 2003.

4. P. Bhandari, M. Prina, R. C. Bowman, C. Paine, D. Pearson, and A. Nash, "Sorption coolers using a continuous cycle to produce $20 \mathrm{~K}$ for the Planck flight mission," Cryogenics 44, pp. 395-401, June 2004.

5. M. Seiffert, A. Mennella, C. Burigana, N. Mandolesi, M. Bersanelli, P. Meinhold, and P. Lubin, "1/f noise and other systematic effects in the Planck-LFI radiometers," A\&BA 391, pp. 1185-1197, Sept. 2002.

6. A. Mennella, M. Bersanelli, M. Seiffert, D. Kettle, N. Roddis, A. Wilkinson, and P. Meinhold, "Offset balancing in pseudo-correlation radiometers for cmb measurements," A\&A 410, pp. 1089-1100, 2003.

7. P. Cappellini, D. Maino, G. Albetti, P. Platania, R. Paladini, A. Mennella, and M. Bersanelli, "Optimized in-flight absolute calibration for extended CMB surveys," A $\mathscr{U} A$ 409, pp. 375-385, 2003.

8. C. Burigana, P. Natoli, N. Vittorio, N. Mandolesi, and M. Bersanelli, "In-Flight Main Beam Reconstruction for Planck-LFI," Experimental Astronomy 12, pp. 87-106, Jan. 2001.

9. M. Bersanelli, "Planck-LFI calibration plan," Tech. Rep. PL-LFI-PST-PL-008, IASF - UniMi, July 2003.

10. A. Mennella, "LFI calibration and testing data analysis methods," Tech. Rep. PL-LFI-PST-TN-068, UniMi, November 2005.

11. M. Tomasi, A. Mennella, P. Battaglia, C. Franceschet, L. Stringhetti, L. Terenzi, F. Cuttaia, M. Lapolla, M. Miccolis, P. Leutenegger, M. Bersanelli, R. Leonardi, P. Meinhold, M. Salmon, M. Sandri, M. Salmon, T. Poutanen, D. Maino, and F. Villa, "Data Analysis of the Planck/LFI Ground-Test Campaign," in Astronomical Telescopes and Instrumentation, Proceedings of the SPIE, 2006. 